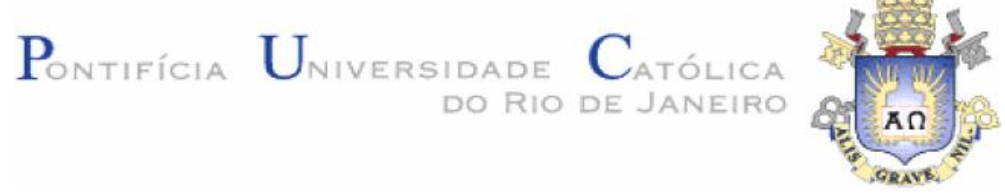

Jose Francisco Consuegra Murgas

\title{
Modelagem Numérica do Ensaio de Leak Off em Poços de Petróleo
}

Dissertação de Mestrado

Dissertação apresentada ao Programa de PósGraduação em Engenharia Civil da PUC-Rio como requisito parcial para obtenção do título de Mestre em Engenharia Civil.

Orientador: Prof. Sergio Augusto Barreto da Fontoura Co-Orientador: Dr. Nelson Inoue

Rio de Janeiro, novembro de 2011. 

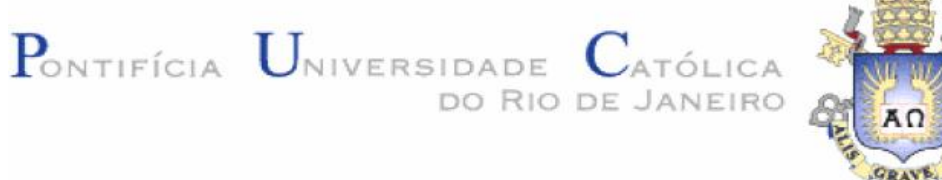

Jose Francisco Consuegra Murgas

\title{
Modelagem Numérica do Ensaio de Leak Off em Poços de Petróleo
}

\begin{abstract}
Dissertação apresentada ao Programa de PósGraduação em Engenharia Civil da PUC-Rio como requisito parcial para obtenção do título de Mestre em Engenharia Civil. Aprovada pela Comissão Examinadora abaixo assinada.
\end{abstract}

Prof. Sergio Augusto Barreto da Fontoura Departamento de Engenharia Civil - PUC-Rio

Orientador

Prof. Alberto Sampaio Ferraz Jardim Sayão Departamento de Engenharia Civil - PUC-Rio

Prof. Paulo Couto Universidade Federal do Rio de Janeiro

Prof. José Eugênio Leal

Coordenador Setorial do Centro Técnico Científico - PUC-Rio

Rio de Janeiro, 18 de novembro de 2011. 
Todos os direitos reservados. É proibida a reprodução total ou parcial do trabalho sem autorização da universidade, do autor e do orientador.

\section{Jose Francisco Consuegra Murgas}

Possui graduação em Engenharia Civil da Universidade Industrial de Santander - UIS (Colômbia-2007). Trabalho em um projeto de vulnerabilidade sísmica na região metropolitana de Bucaramanga-Santander, na área geotécnica e de estruturas. Atualmente é estudante de mestrado em engenharia civil na área de geotecnia na PUC-Rio, Brasil. Além disso, está vinculado a um projeto sobre estabilidade de poços de petróleo em rochas salinas.

Ficha Catalográfica

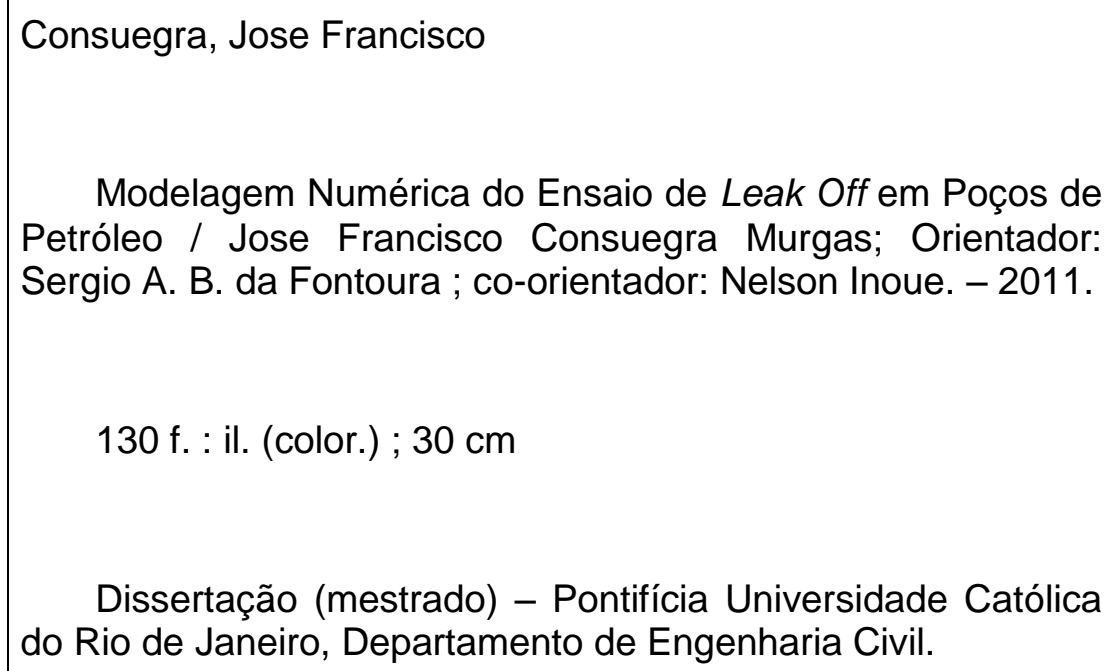

Modelagem Numérica do Ensaio de Leak Off em Poços de Petróleo / Jose Francisco Consuegra Murgas; Orientador: Sergio A. B. da Fontoura ; co-orientador: Nelson Inoue. - 2011.

130 f. : il. (color.) ; $30 \mathrm{~cm}$

Dissertação (mestrado) - Pontifícia Universidade Católica do Rio de Janeiro, Departamento de Engenharia Civil.

Incluí bibliografia.

1. Engenharia Civil - Teses. 2. Leak-Off. 3. Poroelasticidade. 4. Fluência. 5. Permeabilidade. 6. Estabilidade. I. Fontoura, Sergio A. B. da. II. Inoue, Nelson. III. Pontifícia Universidade Católica do Rio de Janeiro. Departamento de Engenharia Civil. IV. Título. 
À memória de minha avó Zaida Murgas, meu eterno amor.

Exemplo de carinho e humildade. 


\section{Agradecimentos}

Á Deus, criador de todas as coisas. Por me permitir escrever um pouco da minha historia e seguir em frente cada dia.

Ao professor Sérgio Fontoura, pela orientação, amizade e confiança conquistada durante o desenvolvimento deste trabalho.

Aos meus pais, Lia e Marcial, pelo amor incondicional, carinho e total apoio durante toda a minha vida. A minha tía Magola minha segunda mãe.

A todos meus irmãos e irmãs. À Nathalia pela paciência, amor e tolerância em toda esta etapa da minha vida.

A toda família, pela bondade, em especial aos meus sobrinhos Daniel e Daniela pelas brincadeiras e risadas.

A todos os meus amigos, de infância, da UIS (Universidade Industrial de Santander), do mestrado (PUC-Rio) e a todas as pessoas que de uma forma ou de outra me estimularam ou me ajudaram.

Ao meu grande amigo Nelson, pela amizade criada, pela troca de conhecimentos e apoio.

À Coordenação de Aperfeiçoamento de Pessoal de Nível Superior (CAPES) pelo apoio financeiro.

Ao Departamento de Engenharia Civil da PUC-Rio, aos professores e aos colegas.

Ao GTEP (Grupo de Tecnologia e Engenharia de Petróleo) pelo apoio técnico durante a dissertação e pela utilização do Abaqus.

Aos professores que participaram da comissão examinadora. 


\section{Resumo}

Consuegra, Jose Francisco; Fontoura, Sergio A. B. da; Inoue, Nelson. Modelagem Numérica do Ensaio de Leak Off em Poços de Petróleo. Rio de Janeiro, 2011. 130p. Dissertação de Mestrado - Departamento de Engenharia Civil, Pontifícia Universidade Católica do Rio de Janeiro.

Em operações de perfuração, é importante ter uma estimativa do gradiente de fratura de determinada formação, como o objetivo de poder estimar o máximo peso da lama necessária para a próxima seção da formação rochosa, antes de colocar o revestimento. Por esse motivo, são realizados ensaios de Leak-Off para determinar a máxima pressão que a nova seção do poço pode sustentar sem fraturar ou perder fluido, sendo realizados durante a fase de perfuração do poço, nas formações imediatamente abaixo de cada sapata de revestimento. A finalidade deste estudo é realizar a simulação numérica deste ensaio usando um programa comercial de elementos finitos para calcular a pressão de Leak-Off, considerando a taxa de bombeamento como um dado de entrada do problema e a pressão na parede do poço como uma resposta. $O$ trabalho abordará dois cenários: uma formação rochosa permeável e uma formação rochosa impermeável constituída de rocha de sal. Deste modo, para um estudo de uma análise real de um ensaio de Leak-Off em rocha permeável e rocha impermeável, torna-se necessário a utilização de modelos que considerem poroelasticidade e fluência, respectivamente. Para a caracterização do comportamento da rocha de sal foi usada uma lei de fluência de duplo mecanismo de deformação, que considera apenas os mecanismos de fluência: planar e indefinido. Os modelos estudados foram bidimensionais e analisados sobre a hipótese de deformação plana. A hipótese, de que a pressão de LeakOff é atingida quando a tensão tangencial efetiva é igual a zero (calculada graficamente), foi considerada. Finalmente, foram simulados alguns casos para verificar os possíveis efeitos da alteração da permeabilidade e influência do fluido de pressurização no cálculo da pressão de Leak-Off em rocha permeável.

\section{Palavras-chave}

Leak-Off, Elementos Finitos; Poroelasticidade; Fluência; Permeabilidade. 


\section{Abstract}

Consuegra, Jose Francisco; Fontoura, Sergio A. B. da (Advisor); Inoue, Nelson (Co-Advisor). Numerical Modeling of Leak Off Test in Oil Wells. Rio de Janeiro, 2011. 130p. MSc. Dissertation - Departamento de Engenharia Civil, Pontifícia Universidade Católica do Rio de Janeiro.

In drilling operations, it is important to have an estimate of the fracture gradient of certain formation, in order to being able to estimate the maximum mud weight required for the next section of the rock formation, before casing is placed. For this reason, Leak-Off tests are conducted to determine the maximum pressure that the new section of the well can support without fracturing or losing fluid, being made during the drilling phase of the well in formations immediately below each casing shoe. The purpose this study is to perform the numerical simulation of this test using a commercial finite element program to calculate the Leak-Off pressure, considering the pumping rate as an input data of the problem and pressure at the borehole wall as the answer. The work will address two cases: a permeable rock formation and an impermeable rock formation composed of salt rock. Thus, for studying a real analysis of a Leak-Off test in permeable and impermeable rock, it becomes necessary to use models that consider poroelasticity and creep, respectively. For the characterization of salt rock it was used a creep law of dual mechanism of deformation, which considers only the dislocation creep mechanisms: planar and undefined. The twodimensional models were studied and analyzed under the assumption of plane strain. The hypothesis that the Leak-Off pressure is reached when the effective tangential stress is zero (estimated graphically), was considered. Finally, some cases were simulated to check the possible effects of changing the permeability and the influence of pressurized fluid in the calculation of Leak-Off pressure in the permeable rock.

\section{Keywords}

Leak-Off; Finite Elements; Poroelasticity; Creep; Permeability. 


\section{Sumário}

1 INTRODUÇÃO 20

1.1. Antecedentes 20

1.2. Objetivo do Trabalho 21

1.3. Escopo do Trabalho 22

2 REVISÃO BIBLIOGRÁFICA 24

2.1. Introdução 24

2.2. O Ensaio de Leak-Off 24

2.2.1. Metodologia do Ensaio de Leak-Off 25

2.2.1.1. Diretrizes de Bombeamento 27

2.2.1.2. Diretrizes de Interpretação 28

2.2.2. Fatores que afetam o ensaio de Leak-Off 29

2.2.2.1. Propriedades do Fluido 29

2.2.2.2. Penetração de Fluidos 29

2.2.2.3. Permeabilidade 30

2.2.2.4. Rocha e Elasticidade 30

2.2.2.5. Efeito do Poço 30

2.2.2.6. Fissuras pré-existentes 31

2.3. Modelagem Numérica e Experimental do Ensaio de Leak-Off 33

3 MODELOS CONSTITUTIVOS 42

3.1. Introdução 42

3.2. Resultados de Ensaios de Compressão Hidrostática e Triaxial 42

3.3. Região Elástica 45

3.4. Região de Endurecimento (Hardening) 45

3.5. Região de Amolecimento (Softening) 46

3.6. Significado da Dilatância 47

3.7. Comportamento da Rocha de Sal $\quad 47$

3.7.1. Variação das Deformações no Tempo 48

3.7.2. Modelo Constitutivo para a Rocha de Sal 49

3.7.3. Ensaios Experimentais e Resultados 50

3.8. Variação da Permeabilidade 51

3.8.1. Antecedentes 51 
3.8.2.1. Equação de Carman-Kozeny em Função da Área de Superfície Específica 55

3.8.2.2. Equação de Carman-Kozeny para Grãos Esféricos Uniformes 57

3.8.2.3. Validação da Equação de Carman-Kozeny 59

3.8.3. Relação da Permeabilidade Absoluta com o Coeficiente de

Condutividade Hidráulica

60

4 POROELASTICIDADE 62

4.1. Introdução 62

4.2. Equações Governantes do Problema de Fluxo 62

4.2.1. Equação da Continuidade 63

4.2.2. Equação de Darcy 64

4.2.3. Equação de Estado 65

4.3. Equações Governantes do Problema de Tensões 69

4.3.1. Equações de Equilíbrio 69

4.3.2. Relações Deformação-Deslocamento 70

4.4. Solução do problema no Abaqus 71

5 MODELAGEM NUMÉRICA E RESULTADOS DO ENSAIO DE LEAK-OFF 73

5.1. Introdução 73

5.2. Simulação Numérica do Ensaio de Leak-Off 74

5.3. Descrição do Problema 76

5.4. Passos Para a Análise 76

$\begin{array}{ll}\text { 5.4.1. Etapa de Equilibrio } & 77\end{array}$

5.4.2. Etapa de Perfuração $\quad 77$

$\begin{array}{ll}\text { 5.4.3. Etapa de Pressurização } & 78\end{array}$

5.4.4. Validação do Uso do Programa Abaqus 78

5.5. Caso 1: Rocha Permeável 81

5.5.1. Malha de Elementos Finitos (Mesh) 82

5.5.2. Análise Poroelástica 84

5.5.2.1. Introdução 84

5.5.2.2. Etapa de Perfuração 84

5.5.2.3. Etapa de Pressurização 86

5.5.3. Análise Poroelástica com Influência de Variação da Permeabilidade 90

5.5.3.1. Introdução 90

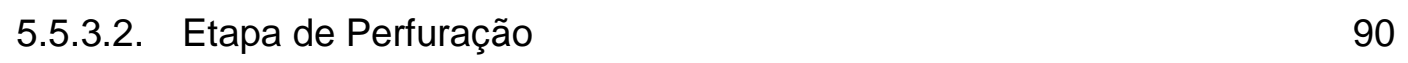


5.5.3.3. Etapa de Pressurização 92

5.5.4. Análise Poroelástica com Influência do Fluido de Pressurização 96

5.5.4.1. Introdução 96

5.5.4.2. Etapa de Perfuração 96

5.5.4.3. Etapa de Pressurização 100

5.6. Caso 2: Rocha de Sal 110

5.6.1. Malha de Elementos Finitos (Mesh) 112

5.6.2. Passos Para a Análise 112

5.6.3. Análise com Comportamento de Fluência 113

5.6.3.1. Introdução 113

5.6.3.2. Etapa de perfuração 114

$\begin{array}{ll}\text { 5.6.3.3. Etapa de Pressurização } & 115\end{array}$

6 CONCLUSÕES E SUGESTÕES PARA TRABALHOS FUTUROS 122

6.1. Conclusões 122

6.2. Sugestões 123

REFERÊNCIAS BIBLIOGRÁFICAS 125 


\section{Lista de Figuras}

Figura 2.1: Linhas guia no ensaio de pressão de integridade - Modificada (Ploster, 1997).

Figura 2.2: Verificação da taxa de bombeamento com as linhas de guia Modificada (Ploster, 1997).

Figura 2.3: Curva típica para um ensaio de Leak-Off - Modificada (Ploster, 1997).

Figura 2.4: Efeito sem fissuras pré-existentes - Modificada (Postler, 1997). 31

Figura 2.5: Efeito de fissuras pré-existentes - Modificada (Postler, 1997). 32

Figura 2.6: Representação do modelo de fratura - Modificada

(Frydman \& Fontoura, 2003).

Figura 2.7: Localização dos elementos auxiliares com o material virtual Modificada (Frydman \& Fontoura, 2003).

Figura 2.8: Curvas de pressão vs. tempo para diferentes valores de viscosidade do fluido - Modificada (Frydman \& Fontoura, 2003).

Figura 2.9: Geometria da fratura para o modelo proposto - Modificada

(Almeida, 1986).

Figura 2.10: Curva de pressão vs. tempo - Modificada (Almeida, 1986).

Figura 2.11: Esquema do conjunto experimental para os ensaios de

Fraturamento hidráulico sob um campo de tensões horizontais anisotropicas, usando um medidor de deformação diametral - Modificada (Zhao et al.,1996). 37 Figura 2.12: Dois registros típicos durante o fraturamento hidráulico no granito Lac du Bonnet usando um medidor de deformação diametral, Campo de tensões $\sigma \mathrm{h}<\sigma \mathrm{H}-$ Modificada (Zhao et al.,1996).

Figura 2.13: Dois registros típicos durante o fraturamento hidráulico no granito Lac du Bonnet usando um medidor de deformação diametral, Campo de tensões $\sigma \mathrm{h}=\sigma \mathrm{H}-$ Modificada (Zhao et al.,1996).

Figura 2.14: Decomposição do (a) o problema original em (b) o problema homogêneo e (c) e (d) dois sub-problemas.

Figura 2.15: Contornos de tensão máxima normalizada para o canto do poço (Golshani \& Tran-Cong, 2006).

Figura 3.1: Deformação sob compressão hidrostática (Goodman, 1989).

Figura 3.2: Deformação sob compressão triaxial: (a) deformação axial e lateral;

(b) dilatância (Goodman, 1989).

Figura 3.3: Idealização bi-linear de ensaio triaxial (Vermeer \& de Borst, 1984). 45 
Figura 3.4: Deslizamento de fissuras em concreto e rocha, e movimento de partículas em solos granulares (Vermeer \& de Borst, 1984). 47

Figura 3.5: Regiões de comportamento em fluência (Goodman, 1989). $\quad 48$

Figura 3.6: Velocidade de um fluido em fluxo laminar - Modificada.

(http://www.spe.org/web/training/demo/mod1/mod1-1.htm).

Figura 3.7: Fluxo de baixa velocidade passando uma partícula - Modificada.

(http://www.spe.org/web/training/demo/mod1/mod1-1.htm).

Figura 3.8: Variação do índice de vazios no tempo, para uma análise poroelástica.

Figura 3.9: Variação da permeabilidade em função do diâmetro dos grãos -

Modificada. (http://www.spe.org/web/training/demo/mod1/mod1-1.htm). 59

Figura 4.1: Fluxo de Fluido Através de um elemento de meio poroso. 63

Figura 5.1: Geometria do modelo, parede do poço e localização onde o fluido

é injetado no material virtual - Modificada. (Inoue \& Fontoura, 2010).

Figura 5.2: Seção horizontal de um poço vertical - Modificada

(Frydman, 2003).

Figura 5.3: Condições iniciais do problema - etapa de equilíbrio. 77

Figura 5.4: Zoom da parede do poço - etapa de perfuração. 78

Figura 5.5: Zoom da parede do poço - etapa de perfuração. 78

Figura 5.6: Elementos usados para a validação do Abaqus com a solução

de Kirsch.

Figura 5.7: Variação das tensões tangenciais e radiais (Kirsch vs. Abaqus) para a análise elástica.

Figura 5.8: Visualização em 3D do modelo da rocha permeável - Modificada. (Inoue \& Fontoura, 2010).

Figura 5.9: Representação de um quarto da malha do poço em 2D.

Figura 5.10: Variação da pressão de poros com o tempo, ao longo do eixo Y. 85

Figura 5.11: Variação do deslocamento com o tempo, ao longo do eixo Y. $\quad 85$

Figura 5.12: (a) Estado de tensão para um tempo, $\mathrm{t}=0 \mathrm{~s}$, e (b) Estado de tensão para um tempo, $\mathrm{t}>0 \mathrm{~s}$.

Figura 5.13: Variação da pressão do fluido no ponto A considerando três taxas de bombeamento.

Figura 5.14: Variação do deslocamento no ponto A considerando três taxas de bombeamento.

Figura 5.15: Pressão de Leak-Off para uma taxa de bombeamento constante de $10.0 \mathrm{gpm}$. 
Figura 5.16: Pressão de Leak-Off para uma taxa de bombeamento constante de $1.0 \mathrm{gpm}$.

Figura 5.17: Pressão de Leak-Off para uma taxa de bombeamento constante de $0.25 \mathrm{gpm}$.

Figura 5.18: Variação da pressão de poros com o tempo, ao longo do eixo Y. 91

Figura 5.19: Variação do deslocamento com o tempo, ao longo do eixo Y.

Figura 5.20: Variação da pressão do fluido no ponto A considerando três taxas de bombeamento.

Figura 5.21: Variação do deslocamento no ponto A considerando três taxas de bombeamento.

Figura 5.22: Pressão de Leak-Off para uma taxa de bombeamento constante de $10.0 \mathrm{gpm}$.

Figura 5.23: Pressão de Leak-Off para uma taxa de bombeamento constante de $1.0 \mathrm{gpm}$.

Figura 5.24: Pressão de Leak-Off para uma taxa de bombeamento constante de $0.25 \mathrm{gpm}$.

Figura 5.25: Variação da pressão de poros com o tempo, ao longo do eixo $Y$ (Fluido A).

Figura 5.26: Variação do deslocamento com o tempo, ao longo do eixo $\mathrm{Y}$ (Fluido A).

Figura 5.27: Variação da pressão de poros com o tempo, ao longo do eixo $Y$ (Fluido B).

Figura 5.28: Variação do deslocamento com o tempo, ao longo do eixo $Y$ (Fluido B).

Figura 5.29: Variação da pressão de poros com o tempo, ao longo do eixo $Y$ (Fluido C).

Figura 5.30: Variação do deslocamento com o tempo, ao longo do eixo $Y$ (Fluido C).

Figura 5.31: Variação da pressão do fluido no ponto A, para três taxas de bombeamento e três fluidos de pressurização (Fluidos $A, B$ e $C$ ).

Figura 5.32: Variação do deslocamento no ponto $A$, para três taxas de bombeamento e três fluidos de pressurização (Fluidos $A, B$ e $C$ ).

Figura 5.33: Pressão de Leak-Off para uma taxa de bombeamento constante de 10.0 gpm (Fluido A).

Figura 5.34: Pressão de Leak-Off para uma taxa de bombeamento constante de 10.0 gpm (Fluido B). 
Figura 5.35: Pressão de Leak-Off para uma taxa de bombeamento constante de 10.0 gpm (Fluido C).

Figura 5.36: Pressão de Leak-Off para uma taxa de bombeamento constante de $1.0 \mathrm{gpm}$ (Fluido A).

Figura 5.37: Pressão de Leak-Off para uma taxa de bombeamento constante de $1.0 \mathrm{gpm}$ (Fluido B).

Figura 5.38: Pressão de Leak-Off para uma taxa de bombeamento constante de $1.0 \mathrm{gpm}$ (Fluido $\mathrm{C}$ ).

Figura 5.39: Pressão de Leak-Off para uma taxa de bombeamento constante de $0.25 \mathrm{gpm}$ (Fluido A).

Figura 5.40: Pressão de Leak-Off para uma taxa de bombeamento constante de $0.25 \mathrm{gpm}$ (Fluido B).

Figura 5.41: Pressão de Leak-Off para uma taxa de bombeamento constante de $0.25 \mathrm{gpm}$ (Fluido $\mathrm{C}$ ).

Figura 5.42: Comparação da pressão de Leak-Off para uma taxa de bombeamento constante de $10.0 \mathrm{gpm}$ e três fluidos de pressurização (Fluidos A, B e C).

Figura 5.44: Visualização em 3D do modelo da rocha de sal - Modificada. (Inoue e Fontoura, 2010).

Figura 5.45: Comparação entre as malhas com e sem elementos virtuais, e sem pressão prescrita (sem lama).

Figura 5.46: Comparação entre as malhas com e sem elementos virtuais, e com pressão prescrita (com lama).

Figura 5.47: (a) Malha com elementos virtuais e (b) Malha sem elementos virtuais.

Figura 5.48: Variação da pressão do fluido no ponto A considerando três taxas de bombeamento.

Figura 5.49: Variação do deslocamento no ponto A considerando três taxas de bombeamento.

Figura 5.50: Variação das tensões e pressão de fluido ao longo do tempo, considerando uma taxa de bombeamento constante de $10.0 \mathrm{gpm}$.

Figura 5.51: Variação das tensões e pressão de fluido ao longo do tempo, considerando uma taxa de bombeamento constante de $1.0 \mathrm{gpm}$.

Figura 5.52: Variação das tensões e pressão de fluido ao longo do tempo, considerando uma taxa de bombeamento constante de $0.25 \mathrm{gpm}$. 
Figura 5.53: Pressão de Leak-Off para uma taxa de bombeamento constante de $10.0 \mathrm{gpm}$.

Figura 5.54: Pressão de Leak-Off para uma taxa de bombeamento constante de $1.0 \mathrm{gpm}$.

Figura 5.55: Pressão de Leak-Off para uma taxa de bombeamento constante de $0.25 \mathrm{gpm}$. 


\section{Lista de Tabelas}

Tabela 3.1: Área de superfície específica típica para areias e argilas

57

Tabela 3.2: Parâmetros usados na equação de variação de permeabilidade 59

Tabela 3.3: Coeficiente de permeabilidade para diferentes tipos de rochas 61

Tabela 5.1: Tensão de sobrecarga $\sigma_{z}$ (Rocha Permeável) 81

Tabela 5.2: Propriedades do fluido de pressurização (Rocha Permeável) 81

Tabela 5.3: Propriedades da rocha permeável. 84

Tabela 5.4: Comparação da pressão de Leak-Off considerando a variação da permeabilidade. 95

Tabela 5.5: Propriedades dos fluidos de pressurização. 96

Tabela 5.6: Comparação da pressão de Leak-Off usando vários fluidos

de pressurização. 109

Tabela 5.7: Tensão de sobrecarga $\sigma_{z}$ (Rocha de Sal) 110

Tabela 5.8: Propriedades do fluido de pressurização (Rocha de Sal). 110

Tabela 5.9: Propriedades da rocha de sal (Halita) 113 


\section{Lista de Símbolos}

$A \quad$ Área transversal

a Raio do poço

$\alpha \quad$ Coeficiente de Biot

$\mathrm{a}_{v} \quad$ Área de superfície específica (área/volume de rocha)

B' Matriz que permite o acoplamento

$c_{k} \quad$ Parâmetro empírico (equação de Carman-Kozeny)

D Matriz de relação tensão-deformação secante

$D_{p} \quad$ Diâmetro do grão ou da partícula

$\mathbf{D}_{\mathbf{T}}$ Matriz de relação tensão-deformação tangencial

E Modulo de Young

$e \quad$ Índice de vazios

$\mathrm{e}_{0} \quad$ Índice de vazios inicial

$\mathrm{e}_{f} \quad$ Índice de vazios final

$\varepsilon_{1} \quad$ Deformação Axial

$\dot{\varepsilon} \quad$ Taxa de deformação devido à fluência na condição de equilíbrio

$\dot{\varepsilon}_{0} \quad$ Taxa de deformação de referência devido à fluência (estado estacionário)

$\dot{\varepsilon}_{\mathrm{v}}^{\mathrm{p}} \quad$ Taxa de expansão volumétrica plástica

$\varepsilon_{v}{ }^{\text {in }}$ Expansão volumétrica plástica do material

$\dot{\varepsilon}_{1}{ }^{\mathrm{p}} \quad$ Taxa de deformação axial plástica

$\varepsilon_{i j} \quad$ Componentes do tensor das deformações

$f_{t} \quad$ Resistência à tração da rocha

G Módulo de cisalhamento

$g \quad$ Aceleração da gravidade

$h \quad$ Altura da fratura

$\mathrm{H}^{\prime} \quad$ Matriz de fluxo

$k \quad$ Permeabilidade absoluta

K Módulo de deformação volumétrica drenado do meio poroso

$\bar{k} \quad$ Coeficiente de condutividade hidráulica

$\mathrm{K}^{\prime} \quad$ Matriz de rigidez

$\mathrm{k}_{\mathrm{f}} \quad$ Modulo de bulk do fluido

$\mathrm{k}_{\mathrm{s}} \quad$ Permeabilidade do meio poroso na direção do fluxo 
L Comprimento da amostra

$\mathrm{L}(\mathrm{t}) \quad$ Comprimento da fratura

Lt Comprimento de um tubo capilar horizontal

$\mathrm{L}^{\prime} \quad$ Matriz que permite o acoplamento

m Vetor igual à unidade para as tensões normais e zero para as cisalhantes

$n \quad$ Coeficiente de tensão (Lei de Fluência)

P Pressão de fluido

p Pressão confinante

$\mathrm{p}_{\mathrm{o}} \quad$ Pressão de poros na formação

$\rho \quad$ Densidade ou massa específica do fluido

$\rho_{0} \quad$ Massa específica do fluido em um instante de referência

$\mathrm{p}_{\mathrm{w}}$ Pressão (ou peso) da lama hidrostática

q Vazão (Capítulo 3)

$q \quad$ Tensão desviadora (Capítulo 5)

$Q \quad$ Energia de ativação

$R \quad$ Constante universal dos gases

Rt Raio de um tubo capilar horizontal

$r \quad$ Distância a partir do eixo do poço

$S \quad$ Superfície específica dos poros

S Tensor das tensões desviadoras

$T \quad$ Temperatura da rocha

$T_{0} \quad$ Temperatura de referência

$\tau \quad$ Tortuosidade (comprimento médio do trajeto / comprimento total) ${ }^{2}$

U1 Deslocamento na direção $x$

U2 Deslocamento na direção y

$\mathrm{u}, \mathrm{v}, \mathrm{w}$ Componentes do vetor dos deslocamentos

$\mathrm{V}_{\mathrm{S}} \quad$ Velocidade aparente do fluido na direção do fluxo

$V_{t} \quad$ Volume total do elemento

$\mathrm{V}_{\mathrm{p}} \quad$ Volume dos poros

$\mathrm{V}_{\mathrm{x}}, \mathrm{V}_{\mathrm{y}}, \mathrm{V}_{\mathrm{y}}$ Velocidades de Darcy nas direções $\mathrm{x}, \mathrm{y}, \mathrm{z}$.

$w \quad$ Abertura da fratura

$\mathrm{w}_{\text {MAX }}$ Abertura maxima da fratura

$\sigma \quad$ Tensão total

$\sigma_{H} \quad$ Tensão horizontal maior 
$\sigma_{h} \quad$ Tensão horizontal menor

$\sigma^{\prime} \quad$ Tensão efetiva

$\sigma_{e f} \quad$ Tensão efetiva de fluência

$\sigma_{0} \quad$ Tensão efetiva de referência

$\sigma_{\mathrm{x}}, \sigma_{\mathrm{y}}, \sigma_{\mathrm{z}}$ Tensão total na direção x, y e z

$\sigma_{\theta} \quad$ Tensão tangencial efetiva

$\sigma_{\theta, \max }$ Tensão tangencial máxima na parede do poço

$\sigma_{\theta, \min }$ Tensão tangencial mínima na parede do poço

$\sigma_{r} \quad$ Tensão radial

$\sigma_{1} \quad$ Tensão principal maior

$\sigma_{3}^{\prime}$ Tensão principal menor na parede do poço

$\sigma_{i j} \quad$ Componentes do tensor das tensões totais

$\Delta P \quad$ Diferença de pressão aplicada na amostra

$\Delta \mathrm{V} \quad$ Diferença de volume na amostra

$\Delta \varepsilon_{\mathrm{V}} \quad$ Variação volumétrica

$\Delta \sigma \quad$ Variação da tensão total

$\Delta \phi \quad$ Variação da porosidade

$\checkmark \quad$ Coeficiente de Poisson

$\gamma \quad$ Peso específico do fluido

$\mu \quad$ Viscosidade dinâmica do fluido

$\lambda \quad$ Parâmetro de Lamé

$\Phi \quad$ Potencial de fluxo

$\phi \quad$ Porosidade

$\phi_{0} \quad$ Porosidade em um instante de referência

$\Psi \quad$ Ângulo de dilatância

$\theta \quad$ Ângulo medido no sentido anti-horário do plano x-y a partir do eixo'x' 